\title{
AUTOMORPHISMS OF THE LATTICE OF RECURSIVELY ENUMERABLE SETS: PROMPTLY SIMPLE SETS
}

\author{
PETER CHOLAK, ROD DOWNEY, AND MICHAEL STOB
}

\begin{abstract}
We show that for every coinfinite r.e. set $A$ there is a complete r.e. set $B$ such that $\mathscr{L}^{*}(A) \approx_{\text {eff }} \mathscr{L}^{*}(B)$ and that every promptly simple set is automorphic (in $\mathscr{E}^{*}$ ) to a complete set.
\end{abstract}

\section{INTRODUCTION}

One important program in the study of the structure of $\mathscr{E}$, the lattice of r.e. sets, is determining the relationship between the algebraic structure of a set and the degrees of the sets that share the same structure. There has been a good deal of success in this program. For example, Soare [1982] showed that all low (in fact, all semilow) sets generate principal filters (in $\mathscr{E}^{*}$ ) isomorphic to $\mathscr{E}^{*}$. (The principal filter generated by an r.e. set $A$ is denoted $\mathscr{L}^{*}(A)$.) From the work of Martin [1966], Lachlan [1968], Soare [1974], and Maass [1984], we know that the orbit of a hyperhypersimple (hhsimple) set $H$ only contains sets with high degree and for every high degree, there is a set of that degree in the orbit of $H$. In this paper, we will consider the interesting subprogram of finding out just what sets are automorphic to a complete set.

This program grew out of Post's Problem. Post's Problem is the question of whether there are more than two r.e. degrees. As we all know, the answer is yes by Friedberg-Muchnik. When Post posed the above question, he also indirectly suggested a program for solving the problem. Post's Program is to find some definable property on $A$ such that if $A$ satisfies this property then $A$ is incomplete and nonrecursive. He suggested that some sort of "thinness" property such as hhsimplicity might work. However, Yates [1965] constructed a complete maximal set, so we know hhsimplicity will not work. Marchenkov [1976] showed that if $A$ is semirecursive and $\eta$-maximal then $A$ is incomplete (see Odifreddi [1989] for details). However, Harrington and Soare have observed that for every $\eta$, the property of being semirecursive, nonrecursive, and $\eta$-maximal is not a definable property in $\mathscr{E}$. Recently, Harrington and Soare

Received by the editors April 11, 1990.

1980 Mathematics Subject Classification (1985 Revision). Primary 03D25.

Key words and phrases. Automorphism, recursively enumerable, promptly simple.

This research was conducted while the first author was a student associate and the other authors were members of the Mathematical Sciences Research Institute (MSRI). The first author was supported by a Department of Education Fellowship. The second author was partially supported by VUW IGC grant, a U.S./N.Z. binational grant, and MSRI. The third author was partially supported by NSF Grant DMS 88-00030, a U.S./N.Z. binational grant, and MSRI. 
[1991] have completed Post's Program by showing that there is a definable property $Q(A)$ in $\mathscr{E}$ such that every r.e. set $A$ satisfying $Q(A)$ is incomplete, and furthermore, there is a simple set $A$ satisfying $Q(A)$. However, it is still open as to what r.e. sets are automorphic to a complete set.

The following partial results are known. As indicated in the first paragraph, all hhsimple sets are automorphic to a complete set (for more on this see $\S 3)$. Downey and Stob [199?] have shown that every low 2 simple set, ev-

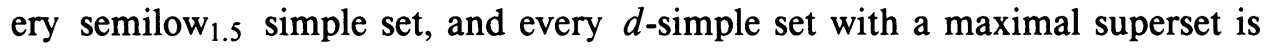
automorphic to a complete set.

In $\S 2$, we prove that for every coinfinite r.e. set $A$ there is a complete r.e. set $B$ such that $\mathscr{L}^{*}(A) \approx_{\text {eff }} \mathscr{L}^{*}(B)$. Hence, in contrast to Harrington and Soare's solution to Post Program, there is no definable property in $\mathscr{E}$ involving only the complement of a set which ensures the set is incomplete. One version of the proof (two different versions appear in $\S 3$ ) involves combining lowness properties with the notion of introreducible sets and then using the result of Soare that if $A$ is semilow then $\mathscr{L}^{*}(A) \approx \mathscr{E}^{*}$.

The main result of this paper is that every promptly simple set is automorphic to a complete set. The proof, which appears in $\S 3$, relies heavily on Soare's Extension Theorem. In this section, we also develop a technical theorem, which when augmented by the Extension Theorem provides us with a different proof of the result in $\S 2$. This technical theorem turns out to be a very useful tool (see Cholak [199?] or Cholak [1991] for more).

We will assume that the reader is familiar with the Extension Theorem of Soare. The best reference on the Extension Theorem is Soare [1987, XV4.6]. For another reference on the result about semilow sets, see Maass [1983]. Our notation is standard and follows Soare [1987]. All sets and degrees used are r.e. except for the set $X$, which is used only in the definitions in $\S 2$. A good reference for the definitions used in $\S 2$ and some additional theorems involving them is Odifreddi [1989]. All other definitions can be found in Soare [1987].

One last note: we only need to consider automorphisms of $\mathscr{E}^{*}$ since, by Soare [1974] and [1987, XV.2], if $\Phi\left(A^{*}\right)=B^{*}$, where $\Phi \in \operatorname{Aut}\left(\mathscr{E}^{*}\right)$ and $A$ is coinfinite and infinite, then there is an automorphism of $\mathscr{E}$ which takes $A$ to $B$.

\section{COMPLETE UNIFORMLY INTROREDUCIBLE SETS}

Definition 2.1 (Jockusch [1968]). A set $X$ is uniformly introreducible if there exists a number $e$ such that $\{e\}^{B}=X$ for all infinite subsets $B$ of $X$.

Definition 2.2 (Jockusch [1968]). A set $X$ is uniformly majorreducible if there is a number $e$ such that for all $f$ if for every $n, f(n) \geq p_{X}(n)$, then $\{e\}^{f}=p_{X}$. (If $X=\left\{x_{0}<x_{1}<x_{2} \cdots\right\}, p_{X}(n)=x_{n}$ is the principal function of $X$.)

It is easy to see that every uniformly majorreducible set is uniformly introreducible. By Jockusch [1968, 6.2], we know that if $\operatorname{deg}(X) \leq \mathbf{0}^{\prime}$ and $p_{X}$ dominates every partial recursive function, then $X$ is uniformly majorreducible and, in fact, $\operatorname{deg}(X)=\mathbf{0}^{\prime}$. The following two theorems are the backbone of this section. 
Theorem 2.3. There exists an r.e. set $A$ such that

(1) $A$ is complete,

(2) $\bar{A}$ is uniformly majorreducible,

(3) $\bar{A}$ is semilow.

(We will prove this theorem below.)

Theorem 2.4. For every coinfinite r.e. $B$, there is a complete r.e. set $C$ such that $\mathscr{L}^{*}(B) \approx_{\text {eff }} \mathscr{L}^{*}(C)$.

Proof of 2.4. Let $A$ be as in Theorem 2.3. By Soare [1982], we know $\mathscr{E} * \approx_{\text {eff }}$ $\mathscr{L}^{*}(A)$ by some isomorphism $\Phi$. Let $C=\Phi(B)$ and define $\Psi$ by

$$
\Psi\left(W_{e} \cup B\right)=\Phi\left(W_{e}\right) \cup C .
$$

Since $\Phi$ is an effective isomorphism, it is easy to see that $\Psi$ is an effective isomorphism from $\mathscr{L}^{*}(B)$ to $\mathscr{L}^{*}(C)$. Since $\bar{C} \subseteq \bar{A}$ for all $x, p_{\bar{C}}(x) \geq p_{\bar{A}}(x)$. Since $\bar{A}$ is uniformly majorreducible, we know that $\operatorname{deg}(A) \leq \operatorname{deg}(C)$ and hence $C$ is complete.

Note that it is not possible to extend this line of reasoning to show that for all $B$ and for all degrees $\mathbf{d}$, if $\mathbf{d}$ is incomplete, then there exists a $C$ such that $\operatorname{deg}(C)=\mathbf{d}$ and $\mathscr{L}^{*}(B) \approx_{\text {eff }} \mathscr{L}^{*}(C)$. To be able to do this using the above line of reasoning, we would need to build an r.e. set $A$ such that $\operatorname{deg}(A)=\mathrm{d}, \bar{A}$ is semilow, and every coinfinite r.e. superset of $A$ has degree d. Unfortunately, this cannot be done since either $A$ is hhsimple and hence $\bar{A}$ cannot be semilow (because, by Lachlan [1968] (see Soare [1987, X.2.8]), $\mathscr{L}^{*}(A)$ is a Boolean algebra and therefore, by Soare, [1982], $\bar{A}$ cannot be semilow) or $A$ is not hhsimple and therefore for all $\mathbf{d}_{1} \geq \operatorname{deg}(A)$ there is an r.e. set $B \supseteq A$ of degree d $_{1}$. (This is due to Lachlan [1968, Theorem 1]; an argument is as follows: Since $A$ is not hhsimple, there exists a weak disjoint array such that $W_{f(e)}$ only contains numbers greater than $e$ and $\left|W_{f(e)} \cap \bar{A}\right|=1$. Let $\mathbf{d}_{\mathbf{1}} \geq \operatorname{deg}(A)$ and $D \in \mathrm{d}_{1}$ be an r.e. set. Define $B_{s}=A_{s} \cup W_{f(e), s}$, if $e \in D_{s}-D_{s-1}$; otherwise, let $B_{s}=A_{s}$. Let $B=\bigcup B_{s}$. It is easy to see that $B$ has degree $\mathrm{d}_{\mathbf{1}}$.) However, if $B$ is not simple, using a similar line of reasoning it is possible to show the following theorem:

Theorem 2.5. If $B$ is not simple but coinfinite and $\mathbf{d} \geq \operatorname{deg}(B)$ then there exists an r.e. set $C \in \mathbf{d}$ with $\mathscr{L}^{*}(B) \approx_{\mathrm{eff}} \mathscr{L}^{*}(C)$.

Proof. Let $R \subseteq \bar{B}$ be an infinite recursive set. Let $f$ be a recursive 1-1 function whose range is $R . \mathscr{E}^{*}(R) \approx_{\mathrm{eff}} \mathscr{E}^{*}$ by $\Upsilon\left(W_{e} \cap R\right)=f^{-1}\left(W_{e}\right)$. $\left(\mathscr{E}^{*}(A)\right.$ denotes the principal ideal generated by the r.e. set $A$ in $\mathscr{E}^{*}$.) Let $D$ be an r.e. set such that $D \in \mathbf{d}$ and $D$ is semilow (see Soare [1987, IV.4.11]). We know $\mathscr{E}^{*} \approx_{\text {eff }} \mathscr{L}^{*}(D)$ by some effective isomorphism $\Phi$. Let $C=B \cup \Upsilon^{-1}(D)$ (since $R$ is recursive and $\mathbf{d} \geq \operatorname{deg}(B), C$ has degree d $)$. Define $\Psi$ by $\Psi\left(W_{e} \cup B\right)=$ $\left[\left(W_{e} \cup B\right) \cap \bar{R}\right] \cup \Upsilon^{-1}\left(\Phi\left(\Psi\left(W_{e} \cap R\right)\right)\right)$. Since $\Upsilon$ and $\Phi$ are effective isomorphisms, it is easy to see that $\Psi$ is an effective isomorphism from $\mathscr{L}^{*}(B)$ to $\mathscr{L}^{*}(C)$.

Proof of 2.3. We will use the following notation: let $\left\{a_{0}^{s}<a_{1}^{s}<a_{2}^{s} \cdots\right\}=\bar{A}_{s}$ and $\left\{a_{0}<a_{1}<a_{2} \cdots\right\}=\bar{A}$. To ensure that $A$ is complete and $\bar{A}$ is uniformly majorreducible, it is enough to meet the following requirements: 
$R_{n}:$ if $\varphi_{e, s}(n) \downarrow$ and $n \geq e$ then $a_{2 n+1} \geq \varphi_{e, s}(n)$.

$N_{e}:|\bar{A}|>e$.

If we meet these requirements, we can show that $A$ is complete and $\bar{A}$ is uniformly majorreducible by the following reasoning. Let $f$ be a 1-1 recursive function whose range is $K$. Define the partial recursive function $\psi(x)=$ $f^{-1}(x)$ if $x \in K$. Since we meet $R_{e}$, for all $e$, there exists a $k$ such that for all $x \geq k$, if $\psi(x) \downarrow$ then $a_{2 x+1} \geq \psi(x)$. To show $\bar{A}$ is uniformly majorreducible suppose $g \geq p_{\bar{A}}$, and define $h(x)=g(2 x+1) \geq a_{2 x+1}$. Therefore for all $x \geq k, x \in K$ iff $x \in\{f(0), f(1), \ldots, f(h(x))\} . h$ is uniformly recursive in $g$. Hence $K$ and therefore $A$ are both uniformly recursive in $g$. (The above is almost the same as the proof of Jockusch [1968, 6.2].) $A$ is complete since $p_{\bar{A}}$ is recursive in $A$ and $p_{\bar{A}}$ computes $K$.

To make $\bar{A}$ semilow, we need to guarantee that $\left\{e: W_{e} \cap \bar{A} \neq \varnothing\right\} \leq_{\mathrm{T}} \mathbf{0}^{\prime}$. We $e$-tag $x$ at stage $s+1$ if $x$ is the least $y$ such that $y \in W_{e, s} \cap \bar{A}_{s}$ and there are no $e$-tagged elements of $\bar{A}_{s}$. If $x$ is $e$-tagged we will only allow requirements of higher priority, $R_{k}$ for $k \leq e$, to put $x$ into $A$.

Stage $s=0$. Define $A_{0}=\varnothing$.

Stage $s+1$. Let $n$ be the least $x$ such that there exists a $k \leq x$ with $a_{2 x+1}^{s}<\varphi_{k, s}(x) \downarrow$. For all $m$, if $a_{n}^{s}<a_{m}^{s} \leq s$ and $a_{m}^{s}$ is not $j$-tagged for any $j<n$, enumerate $a_{m}^{s}$ into $A$ at stage $s+1$. Since there are at most $n$ elements of $\bar{A}_{s}$ that are $j$-tagged for $j<n$, we have that $a_{2 n+1}^{s+1}>s \geq \varphi_{k, s}(n) \downarrow$.

It is clear that $R_{n}$ acts only a finite number of times and that $N_{e}$ is met. It only remains to show $\bar{A}$ is semilow.

Lemma 2.6. $\bar{A}$ is semilow.

Proof. Fix $e$. Using $K$ find $s$ such that if $\varphi_{k}(n) \downarrow$ then $\varphi_{k, s}(n) \downarrow$ for $n, k \leq$ $e$. We know that $R_{n}$, for $n \leq e$, will not act after $s+e+1$. If $x \in \bar{A}_{s+e+1}$ is $e$ tagged or $x$ is $e$-tagged after stage $s+e+1$ then $x \in \bar{A}$ and hence $W_{e} \cap \bar{A} \neq \varnothing$. Using $K$ we determine whether such an element exists. $W_{e} \cap \bar{A} \neq \varnothing$ if and only if there exists one $e$-tagged element of $\bar{A}$. $\square$.

The proof of Theorem 2.3 is a modified "dump" construction. If we could use the normal "dump" construction (i.e. for every $m$ such that $a_{n}^{s}<a_{m}^{s} \leq$ $s$, enumerate $a_{m}^{s}$ into $A$ ), we could make $\bar{A}$ retraceable. However by the following theorem we cannot do this.

Definition 2.7. (i) $X$ is retraceable if there exists a partial recursive function $\varphi$ such that $\varphi\left(x_{n+1}\right)=x_{n}$ and $\varphi\left(x_{0}\right)=x_{0}$, where $X=\left\{x_{0}<x_{1}<x_{2} \cdots\right\}$.

(ii) $X$ is regressive if there exist a partial recursive function $\varphi$ and some fixed listing of $X=\left\{x_{0}, x_{1}, x_{2}, \ldots\right\}$ such that $\varphi\left(x_{n+1}\right)=x_{n}$ and $\varphi\left(x_{0}\right)=x_{0}$.

Theorem 2.8. There is no r.e. set $A$ such that

(1) $A$ is complete,

(2) $\bar{A}$ is regressive,

(3) $\bar{A}$ is semilow. 
Proof. Assume $A$ is an r.e. complete set with $\bar{A}$ regressive and semilow. Using $A$ we will build two r.e. sets $B$ and $C$. Since $A$ is complete, by using the Recursion Theorem, we can assume $B=\Phi_{e}^{A}$ and $C=W_{i}$, for some $e$ and $i$. Let $g$ be the characteristic function of the set $\left\{e: W_{e} \cap \bar{A} \neq \varnothing\right\}$. By the Limit Lemma, $g(x)=\lim _{s \rightarrow \omega} f(x, s)$ where $f$ is recursive. Since $\bar{A}$ is regressive, there exists a partial recursive function $\varphi$ such that for some fixed listing of $\bar{A}=\left\{a_{0}, a_{1}, a_{2}, \ldots\right\}, \varphi\left(a_{n+1}\right)=a_{n}$ and $\varphi\left(a_{0}\right)=a_{0}$.

We say $y$ is ready at stage $t$ if $\Phi_{e, t}^{A_{t}}(y)=0$, there exist a subset $\hat{A}_{t} \subseteq \bar{A}_{t}$, a listing of $\hat{A}_{t}=\left\{a_{0}^{t}, a_{1}^{t}, a_{2}^{t} \cdots\right\}$ (note we are not assuming that $a_{i}^{t}<a_{i+1}^{t}$; this is different than the use of this notation in the proof of Theorem 2.3) and $k_{t}$ such that:

(i) for all $z<u\left(A_{t} ; e, y, t\right), z \in A_{t}$ or $z=a_{k}^{t}$ for some $k<k_{t}$,

(ii) $a_{k_{t}}^{t}>u\left(A_{t} ; e, y, t\right)$,

(iii) for all $k<k_{t}, \varphi\left(a_{k+1}^{t}\right)=a_{k}^{t}$, and

(iv) $\varphi\left(a_{0}^{t}\right)=a_{0}^{t}$.

Let $k(y, t)$ be the least such $k_{t}$ and $a(y, t)=a_{k(y, t)}^{t}$.

Assume $y$ is ready at stage $s$. For any $k \leq k(y, s)$, if $a_{k}^{s} \in A$, then for all $j$ with $k \leq j \leq k(y, s), a_{j}^{s} \in A$. At some later stage $t$, if $y$ is not ready at stage $t$ or $a(y, s) \neq a(y, t)$, then $a(y, s) \in A$.

We will say $y$ is started at stage $t$ if $y$ is ready at stage $t$ and $a(y, t) \in C_{t}$. We will build $B$ and $C$ in the following manner.

Stage $s=0$. Define $B_{0}=\varnothing, C_{0}=\varnothing$, and $b_{0}=0$.

Stage $s+1$. Do one of the following:

(i) If $b_{s}$ is not ready at stage $s$ or $b_{s}$ is not started at stage $s$ and $f(i, s)=1$, let $B_{s+1}=B_{s}, C_{s+1}=C_{s}$, and $b_{s+1}=b_{s}$.

(ii) If $b_{s}$ is ready at stage $s$ and $f(i, s)=0$, let $C_{s+1}=C_{s} \cup\left\{a\left(b_{s}, s\right)\right\}$, $B_{s+1}=B_{s}$, and $b_{s+1}=b_{s}$. (So either $b_{s+1}$ is started at stage $s+1$ or for some $k \leq k\left(b_{s}, s\right), a_{k}^{s} \in A_{s+1}$.)

(iii) If $b_{s}$ is started at stage $s$ and $f(i, s)=1$, let $C_{s+1}=C_{s} \cup\left\{a\left(b_{s}, s\right)\right\}$, $B_{s+1}=B_{s} \cup\left\{b_{s}\right\}$, and $b_{s+1}=s+1$.

Now if $b_{s} \in B_{s+1}-B_{s}$ then $b_{s}$ is ready at stage $s$ and for some stage $t>s$, $\Phi_{e, t}^{A_{t}}\left(b_{s}\right)=1$, and hence $a\left(b_{s}, s\right) \notin A$. There exists $t_{1}$ such that for all $s \geq t_{1}$, $f\left(i, t_{1}\right)=f(i, s)$. Since $b_{s}$ can only change at most once after each change in $f(i, s)$, there exist $t_{2} \geq t_{1}$ and $b$ such that for all $s \geq t_{2}, b_{s}=b$. Because $\bar{A}$ is regressive and $A$ is complete, there exists $t_{3} \geq t_{2}$ and $j$ such that $b$ is ready at stage $t_{3}$ and for all $s \geq t_{3}, a\left(b_{s}, s\right)=a_{j}$ (in the above listing of $A$ ). Now if $f\left(i, t_{3}\right)=0,\left\{a_{j}\right\}=C \cap \bar{A}$ and otherwise $\varnothing=C \cap A$.

It is possible to extend the proof of 2.8 to show there is no r.e. complete set $A$ such that $\bar{A}$ is both semilow 2 and regressive. Let

$$
\lim _{s \rightarrow \omega} \lim _{t \rightarrow \omega} f(e, s, t)=\left\{e: W_{e} \cap \bar{A} \neq *^{*} \varnothing\right\} .
$$

Now for every $s$, we can beat $f(i, s, t)$ as we beat $f(i, t)$. We will leave the details to the reader. 


\section{Promptly Simple SetS}

Definition 3.0. A coinfinite r.e. set $A$ is promptly simple if there is a recursive function $p$ and an enumeration $\left\{A_{s}\right\}$ of $A$ so that for all $n \in \omega$,

$$
W_{n} \text { is infinite } \Rightarrow \exists s \exists x\left[x \in\left(W_{n, s}-W_{n, s-1}\right) \cap A_{p(s)}\right] .
$$

To show every promptly simple set $A$ is automorphic to a complete set, we will break the proof into parts: one where $A$ is not hhsimple and one where $A$ is hhsimple.

Theorem 3.1. For every high degree $\mathbf{h}$ and every hhsimple set $A$ there is $B \in \mathbf{h}$ such that $A$ is automorphic to $B$.

Proof. By Lachlan [1968] (see Soare [1987, X.2.8]), we know there is a $\Sigma_{3}$ Boolean algebra $\mathscr{B}$ such $\mathscr{L}^{*}(A) \approx_{\Delta_{3}} \mathscr{B}$. Again by Lachlan [1968] (see Soare $[1987, \mathrm{X} .7 .2])$, there is a $B \in \mathbf{h}$ such that $\mathscr{L}^{*}(B) \approx_{\Delta_{3}} \mathscr{B}$. So $\mathscr{L}^{*}(A) \approx_{\Delta_{3}}$ $\mathscr{L}^{*}(B)$. Thus, by Maass [1984], we know $A \approx_{\Delta_{3}} B$.

Theorem 3.2. Every non-hhsimple promptly simple set is effectively automorphic to a complete set.

Proof. Let $A$ be a non-hhsimple promptly simple set. Let $p$ be a recursive function and $\left\{A_{s}\right\}$ an enumeration of $A$ so that for all $n \in \omega$,

$$
W_{n} \text { is infinite } \Rightarrow \exists^{\infty} s \exists^{\infty} x\left[x \in\left(W_{n, s}-W_{n, s-1}\right) \cap A_{p(s)}\right] .
$$

We will build a complete set $B$ and a $\Phi \in \operatorname{Aut}\left(\mathscr{E}^{*}\right)$ such that $\Phi(A)=B$. As in most automorphism constructions, we will fix two copies of the natural numbers $\omega$ and $\hat{\omega}$ (all integers living on the hatted side will wear hats). Now given a simultaneous enumeration of $\left\{W_{n}\right\}_{n<\omega}$ living in $\omega$, say $\left\{U_{n}\right\}_{n<\omega}$ where $U_{0}=A$ and $U_{1}=K$, we will build the image of $U_{n}$ in $\hat{\omega}, \widehat{U}_{n}=\Phi\left(U_{n}\right)$. Given another simultaneous enumeration of $\left\{W_{n}\right\}_{n>\omega}$ living in $\widehat{\omega}$, say $\left\{V_{n}\right\}_{n<\omega}$, we will build the preimage of $V_{n}$ in $\omega, \widehat{V}_{n}=\Phi^{-1}\left(V_{n}\right)$. Thus, we define $B=\widehat{U}_{0}$. To ensure that $\Phi$ is an automorphism, we need to meet the requirements $R_{\nu}$ below. However, to state the requirements, we need the following definitions.

Definition 3.3. Given simultaneous enumerations $\left\{X_{n, s}\right\}_{n, s<\omega}$ and $\left\{Y_{n, s}\right\}_{n, s<\omega}$ of $\left\{X_{n}\right\}_{n<\omega}$ and $\left\{Y_{n}\right\}_{n<\omega}$, define

(i) the full e-state of $x$ at stage $s, \nu(e, x, s)$, with respect to (w.r.t) $\left\{X_{n, s}\right\}_{n, s<\omega}$ and $\left\{Y_{n, s}\right\}_{n, s<\omega}$ to be the triple

$$
\nu(e, x, s)=\langle e, \sigma(e, x, s), \tau(e, x, s)\rangle,
$$

where

$$
\sigma(e, x, s)=\left\{i: i \leq e \& x \in X_{i, s}\right\}
$$

and

$$
\tau(e, x, s)=\left\{i: i \leq e \& x \in Y_{i, s}\right\} .
$$

(ii) the final e-state of $x, \nu(e, x)$, with respect to $\left\{X_{n, s}\right\}_{n, s<\omega}$ and $\left\{Y_{n, s}\right\}_{n, s<\omega}, \nu(e, x)$, to be $\lim _{s \rightarrow \infty} \nu(e, x, s)$.

The requirements are the following:

$$
U_{1}(=K) \leq_{T} B
$$


and for each final $e$-state $\nu$,

$$
\exists^{\infty} x \in \omega \text { with final } e \text {-state } \nu \text { w.r.t. to }\left\{U_{n, s}\right\}_{n, s<\omega} \text { and }\left\{\hat{V}_{n, s}\right\}_{n, s<\omega}
$$

$R_{\nu}:$

iff

$\exists^{\infty} \hat{x} \in \hat{\omega}$ with final $e$-state $\nu$ w.r.t. to $\left\{\widehat{U}_{n, s}\right\}_{n, s<\omega}$ and $\left\{\widehat{V}_{n, s}\right\}_{n, s<\omega}$.

The standard strategy to meet the requirement $\theta$ is to build a functional $\boldsymbol{\theta}$ such that for every $x$ there is a $t_{x}$ such that for all $y \leq x$ and $s \geq t_{x}$, $\Theta_{s}^{B_{s}}(y)=U_{1, s}(y)$. In order to do this, at any stage $s$ we must be able to add elements to $B_{s}$. The easiest possible strategy to meet $R_{\nu}$ is exact matching, that is to take some matching function $m$ (say a recursive permutation) and let $\widehat{U}_{n, s}=m\left(U_{n, s}\right)$ and $\widehat{V}_{n, s}=m^{-1}\left(V_{n, s}\right)$, for all $n, s<\omega$. Since $\operatorname{deg}(m(A))=$ $\operatorname{deg}(A)$, these two strategies conflict. Exact matching will fail on the numbers we add to $B$ to meet the requirement $\Theta$.

We will break the problem of meeting $R_{\nu}$ into two parts. In one part, we will use exact matching, via the identity function, to meet $R_{\nu}$. In the second part, exact matching will not work. Let $T \subseteq \omega$ and $\widetilde{T} \subseteq \hat{\omega}$ be the same r.e. set of integers where it is not possible to use exact matching. $(T=\widetilde{T}$ since the matching function is the identity. We will see why these sets are r.e. later.) So $R_{\nu}$ divides into two subrequirements,

$$
\exists^{\infty} x \in \bar{T} \text { with final } e \text {-state } \nu \text { w.r.t. to }\left\{U_{n, s}\right\}_{n, s<\omega} \text { and }\left\{\widehat{V}_{n, s}\right\}_{n, s<\omega}
$$

$\exists^{\infty} \hat{x} \in \overline{\widetilde{T}}$ with final $e$-state $\nu$ w.r.t. to $\left\{\widehat{U}_{n, s}\right\}_{n, s<\omega}$ and $\left\{V_{n, s}\right\}_{n, s<\omega}$, and

$$
\begin{aligned}
& \exists^{\infty} x \in T \text { with final } e \text {-state } \nu \text { w.r.t. to }\left\{U_{n, s}\right\}_{n, s<\omega} \text { and }\left\{\widehat{V}_{n, s}\right\}_{n, s<\omega} \\
& R_{\nu}^{1}: \quad \text { iff } \\
& \exists^{\infty} \hat{x} \in \widetilde{T} \text { with final } e \text {-state } \nu \text { w.r.t. to }\left\{\widehat{U}_{n, s}\right\}_{n, s<\omega} \text { and }\left\{V_{n, s}\right\}_{n, s<\omega} .
\end{aligned}
$$

We will meet $R_{\nu}^{0}$ by using our exact matching and meet $R_{\nu}^{1}$ by using the Extension Theorem.

To meet the requirement $\theta$, it will be enough to only act at stage $s+1$ if $0=\Theta_{s}^{B_{s}}(x)=U_{1, s}(x) \neq U_{1, s+1}(x)=1$. We will act by adding some $\hat{y}$ to $B$ where $\hat{y} \leq \theta(x, s)$ (the use of $\Theta_{s}^{B_{s}}(x)$ ) which allows us to legally let $\Theta_{s+1}^{B_{s+1}}(x)=1$. Let $\widetilde{T}_{1}$ be the set of numbers added to $B$ for the sake of this requirement. This set will be r.e. and since we can no longer match these numbers, $\widetilde{T}_{1} \subseteq \widetilde{T}$. (More on all of this later.) To meet $R_{\nu}^{1}$, we will be forced to add more elements to $\widetilde{T}$ but we will do this in an r.e. manner (again more on this later). So assume that $\widetilde{T}$ is r.e. and hence that it is possible to use the Extension Theorem. Before we state the Extension Theorem, the following definitions are needed.

Definition 3.4. Given states $\nu=\langle e, \sigma, \tau\rangle$ and $\nu^{\prime}=\left\langle e^{\prime}, \sigma^{\prime}, \tau^{\prime}\right\rangle$, then

(i) $\nu \preceq \nu^{\prime}$ iff $e \leq e^{\prime}, \sigma=\sigma^{\prime} \cap\{0,1, \ldots, e\}$, and $\tau=\tau^{\prime} \cap\{0,1, \ldots, e\}$.

(ii) $\nu^{\prime}$ covers $\nu\left(\nu \leq \nu^{\prime}\right)$ iff $e=e^{\prime}, \sigma \subseteq \sigma^{\prime}$ and $\tau^{\prime} \subseteq \tau$.

(iii) $\nu^{\prime}$ cocovers $\nu$ iff $\nu \geq \nu^{\prime}$. 
Definition 3.5. Given recursive enumerations $\left\{X_{s}\right\}_{s<\omega}$ and $\left\{Y_{s}\right\}_{s<\omega}$ of $X$ and $Y$, then

(i) $X \backslash Y=\left\{z:(\exists s)\left[z \in X_{s}-Y_{s}\right]\right\}$,

(ii) $X \searrow Y=(X \backslash Y) \cap Y$.

Theorem 3.6 (The Extension Theorem) (Soare [1974] or [1987, XV.6]). Assume $T$ and $\widetilde{T}$ are infinite r.e. sets and $\left\{U_{n}\right\}_{n<\omega},\left\{\widetilde{V}_{n}\right\}_{n<\omega},\left\{\widetilde{U}_{n}\right\}_{n<\omega}$, and $\left\{V_{n}\right\}_{n<\omega}$ are recursive arrays of r.e. sets. Let $\left\{T_{s}\right\}_{s<\omega},\left\{\widetilde{T}_{s}\right\}_{s<\omega},\left\{U_{n, s}\right\}_{n, s<\omega},\left\{\widetilde{V}_{n, s}\right\}_{n, s<\omega}$, $\left\{\widetilde{U}_{n, s}\right\}_{n, s<\omega}$, and $\left\{V_{n, s}\right\}_{n, s<\omega}$ be a simultaneous enumeration of the above r.e. sets. For each full e-state $\nu$ define

$D_{\nu}^{T}=\left\{x: x \in T_{s}-T_{s-1}\right.$ and $\nu=\nu(e, x, s)$ w.r.t. $\left\{U_{n, s}\right\}_{n, s<\omega}$ and $\left.\left\{\widetilde{V}_{n, s}\right\}_{n, s<\omega}\right\}$ and

$D_{\nu}^{\widetilde{T}}=\left\{\hat{x}: \hat{x} \in \widetilde{T}_{s}-\widetilde{T}_{s-1}\right.$ and $\nu=\nu(e, \hat{x}, s)$ w.r.t. $\left\{\widetilde{U}_{n, s}\right\}_{n, s<\omega}$ and $\left.\left\{V_{n, s}\right\}_{n, s<\omega}\right\}$. If $x \in D_{\nu}^{T}$, we say that $\nu$ is the entry e-state of $x$ (likewise for $\hat{x}$ ). Suppose our simultaneous enumeration satisfies the conditions below:

$$
\forall n\left[T \searrow \widetilde{V}_{n}=\widetilde{T} \searrow \widetilde{U}_{n}=\varnothing\right],
$$

$(\forall \nu)\left[D_{\nu}^{\widetilde{T}}\right.$ is infinite $\Rightarrow\left(\exists \nu^{\prime} \geq \nu\right)\left[D_{\nu^{\prime}}^{T}\right.$ is infinite $\left.]\right]$,

$$
(\forall \nu)\left[D_{\nu}^{T} \text { is infinite } \Rightarrow\left(\exists \nu^{\prime} \leq \nu\right)\left[D_{\nu^{\prime}}^{\widetilde{T}} \text { is infinite }\right] .\right.
$$

(We will sometimes say that condition (2) is " $T$ covers $\widetilde{T}$ " and condition (3) is “ $\widetilde{T}$ cocovers $T$ ".) Then there are r.e. sets $\hat{U}_{n}$ extending $\widetilde{U}_{n}$ and $\widehat{V}_{n}$ extending $\widetilde{V}_{n}$ such that $R_{\nu}^{1}$ is satisfied. Furthermore, $\widetilde{U}_{n}=\widehat{U}_{n} \backslash \widetilde{T}$ (this follows from the proof since if $x \notin \widetilde{U}_{n}$, we will only add $x$ to $\hat{U}_{n}$ if $x \in \widetilde{T}$ ).

If we have a simultaneous enumeration of $T, \widetilde{T},\left\{U_{n}\right\}_{n<\omega},\left\{\widetilde{V}_{n}\right\}_{n<\omega}$, $\left\{V_{n}\right\}_{n<\omega}$, and $\left\{\widetilde{U}_{n}\right\}_{n<\omega}$ that satisfies the hypotheses of the Extension Theorem and the following requirements

$\exists^{\infty} x \in \bar{T}$ with final $e$-state $\nu$ w.r.t. to $\left\{U_{n, s}\right\}_{n, s<\omega}$ and $\left\{\widetilde{V}_{n, s}\right\}_{n, s<\omega}$

$\widetilde{R}_{\nu}^{0}$ iff

$\exists^{\infty} \hat{x} \in \overline{\widetilde{T}}$ with final $e$-state $\nu$ w.r.t. to $\left\{\tilde{U}_{n, s}\right\}_{n, s<\omega}$ and $\left\{V_{n, s}\right\}_{n, s<\omega}$,

then using the Extension Theorem it is possible to meet the $R_{\nu}$. Since $\widetilde{U}_{0}$ and $\widetilde{T} \searrow \widehat{U}_{0}$ form a splitting of $B \quad\left(=\hat{U}_{0}\right)$, to meet the requirement $\Theta$ it is enough to meet the subrequirement $\Gamma$,

$$
U_{1}(=K) \leq_{T} \widetilde{U}_{0}
$$

Assume that $\left\{U_{n, s}\right\}_{n, s<\omega}$ and $\left\{V_{n, s}\right\}_{n, s<\omega}$ are simultaneous enumerations of $\left\{W_{e}\right\}_{e<\omega}$, such that $U_{0}=A, U_{1}=K$, for every stage $s>0$ there exists a unique $\langle x, i\rangle$ such that $x \in U_{i, s}-U_{i, s-1}$, and there exists a unique $\langle\hat{x}, c\rangle$ such that $\hat{x} \in V_{c, s}-V_{c, s-1}$. We need to build a simultaneous enumeration $\left\{T_{s}\right\}_{s<\omega},\left\{\widetilde{T}_{s}\right\}_{s<\omega},\left\{\widetilde{V}_{n, s}\right\}_{n, s<\omega}$, and $\left\{\widetilde{U}_{n, s}\right\}_{n, s<\omega}$ of $T, \widetilde{T},\left\{\widetilde{V}_{n}\right\}_{n<\omega}$, and 
$\left\{\widetilde{U}_{n}\right\}_{n<\omega}$ such that $\Gamma$ and $\widetilde{R}_{\nu}^{0}$ are met and the enumeration satisfies the hypotheses of the Extension Theorem. (Clearly, if desired, the above enumerations can be combined into one simultaneous enumeration which also satisfies the hypotheses of the Extension Theorem.)

The following notation will be very helpful. We will use $\nu(e, x, s)$ for the full $e$-state of $x$ at stage $s$ with respect to $\left\{U_{n, s}\right\}_{n, s<\omega}$ and $\left\{\widetilde{V}_{n, s}\right\}_{n, s<\omega}$ and $\tilde{\nu}(e, \hat{x}, s)$ for the full $e$-state of $\hat{x}$ at stage $s$ with respect to $\left\{\widetilde{U}_{n, s}\right\}_{n, s<\omega}$ and $\left\{V_{n, s}\right\}_{n, s<\omega}$.

We will use exact matching to meet $\widetilde{R}_{\nu}^{0}$. So if $x \in\left(U_{n, s} \cap \bar{T}_{s}\right)-U_{n, s-1}$, we will put $\hat{x}$ into $\widetilde{U}_{n, s}(\hat{x}$ is the copy of $x$ in $\hat{\omega})$ and if $\hat{x} \in\left(V_{n, s} \cap \overline{\widetilde{T}}_{s}\right)-V_{n, s-1}$, we will put $x$ into $\widetilde{V}_{n, s}(x$ is the copy of $\hat{x}$ in $\omega$ ). If we only add numbers to the sets $\widetilde{U}_{n}$ and $\widetilde{V}_{n}$ in this fashion we will meet condition (1) of the Extension Theorem. Clearly, as long as $T=\widetilde{T}$, this action will meet $\widetilde{R}_{\nu}^{0}$.

To meet the subrequirement $\Gamma$, we will build a functional $\Gamma$ such that $\Gamma^{\widetilde{U}_{0}}=$ $U_{1}$. Let $\gamma(x, s)$ be the use of $\Gamma_{s}^{\widetilde{U}_{0, s}}(x)$ if $\Gamma_{s}^{\widetilde{U}_{0, s}}(x) \downarrow$ and $\gamma(x, s) \uparrow$ otherwise. We wish to have $\lim _{s \rightarrow \infty} \Gamma_{s}^{\widetilde{U}_{0, s}}(x)=U_{1, s}(x)$. If $0=\Gamma_{s}^{\widetilde{U}_{0, s}}(x)=U_{1, s}(x) \neq$ $U_{1, s+1}(x)=1$, we must add some $\hat{y} \leq \gamma(x, s)$ to $\widetilde{U}_{0, s+1}$, so we can legally let $\Gamma_{s+1}^{\widetilde{U}_{0, s+1}}(x)=1$. $\widetilde{T}_{1}$ will be the set of integers added to $\widetilde{U}_{0}$ for the sake of requirement $\Gamma$. Since, when we add $\hat{y}$ to $\widetilde{U}_{0, s+1}$, it is not always the case that $y \in A_{s+1}$, so we can no longer match $y$ with $\hat{y}$ and hence $\widetilde{T}_{1} \subset \widetilde{T}$. To meet the requirement $\Gamma$ we will act in the following manner.

Since $A$ is not hhsimple, there exists a weak array $\left\{W_{f(i)}\right\}_{i<\omega}$ such that $W_{f(i)}$ only contains numbers greater than $i$ and $\left|W_{f(i)} \cap \bar{A}\right|=2^{2 i+2}+2$. We only need to act at stage $s+1$ if there exists a $z$ such that $0=\Gamma_{s}^{\widetilde{U}_{0, s}}(z)=U_{1, s}(z) \neq$ $U_{1, s+1}(z)=1$. We will act by adding some $\hat{x}$ to $\widetilde{U}_{0, s+1}$ and $\widetilde{T}_{1, s+2}$ (we will build $\widetilde{T}$ slower to meet condition (1)), where $\hat{x} \notin \widetilde{U}_{0, s}, \hat{x} \leq \gamma(z, s)$, and $x \in\left(W_{f(z), s} \cap \bar{A}_{s}\right) \backslash \gamma(z, s)$. We will ensure that if $\gamma(z, s) \downarrow$ then $\left(W_{f(z), s} \cap \bar{A}_{s}\right) \mid$ $\gamma(z, s) \neq \varnothing$ and therefore such a $\hat{x}$ will exist. Hence, if $z \in U_{1, s+1}-U_{1, s}$ and $\gamma(z, s) \downarrow$, it will be possible to act at that stage, if needed.

Meeting conditions (2) and (3) of the hypotheses of the Extension Theorem requires a bit more work. At this point, the only way for $\hat{x}$ to enter $\widetilde{T}$ is through $\widetilde{T}_{1}$ and if $\hat{x}$ enters $\widetilde{T}$ through $\widetilde{T}_{1}$ in state $\nu=\langle e, \sigma, \tau\rangle$, then $0 \in \sigma$. To try to cover this entry state, we will need to use the fact that $A$ is promptly simple. Using the Recursion Theorem, we will build sets $X_{\nu}=W_{g(\nu)}$, for all full $e$-states $\nu$. At stage $s+1$, if we put $\hat{x}$ into $\widetilde{U}_{0, s+1}$ and $\widetilde{T}_{1, s+2}$ (for the sake of subrequirement $\Gamma$ ) in entry state $\nu$, we will also put $x$ into $X_{\nu, s+1}$. While we can no longer match $x$ with $\hat{x}$, we will delay enumerating $x$ into $T$. We let $T$ be the disjoint union of $T_{1}$ and $T_{2}$ defined as follows.

We will wait for a stage $t \geq s+1$ where $x \in W_{g(\nu), t}-W_{g(\nu), t-1}$. At stage $p(t)$, if $x \in A_{p(t)}$, we put $x$ in $T_{1, p(t)}$, otherwise, we put $x$ in $T_{2, p(t)}$. If we put $x$ in $T_{1}$, we will have covered $\hat{x}$. Since $\tilde{\nu}(e, \hat{x}, s)=\nu(e, x, s)=\langle e, \sigma, \tau\rangle$, $\hat{x}$ 's entry state is $\nu(e, x, p(t))=\left\langle e, \sigma^{\prime}, \tau\right\rangle$, where $\sigma \cup\{0\} \subseteq \sigma^{\prime}$ (we will stop matching $x$ and $\hat{x}$ at stage $s$ ). Now if $D_{\nu}^{\widetilde{T}_{1}}$ (defined as above) is infinite, $X_{\nu}$ 
will be infinite and, since $A$ is promptly simple, there exists $\nu^{\prime} \geq \nu$ such that $D_{\nu^{\prime}}^{T_{1}}$ is infinite. Similar reasoning shows that if $D_{\nu}^{T_{1}}$ is infinite then there exists $\nu^{\prime} \leq \nu$ such that $D_{\nu^{\prime}}^{\widetilde{T}_{1}}$ is infinite.

But not all numbers enter $T$ through $T_{1}$. We still need to cocover numbers entering $T$ through $T_{2}$. To do this, we need to make a slight change in the action we use to meet the requirement $\Gamma$. We will ensure that if $\gamma(x, s) \downarrow$ then $\left|\left(W_{f(x), s} \cap \bar{A}_{s}\right)\right| \gamma(x, s) \mid \geq 2^{2 x+2}+2$. If we need to act at stage $s+1$ to meet the subrequirement $\Gamma$ at argument $x$, then we will find $x_{1}, x_{2} \in W_{f(x), s} \cap \bar{A}_{s}$ such that $\nu\left(x, x_{1}, s\right)=\nu\left(x, x_{2}, s\right)=\langle e, \sigma, \tau\rangle$ and $\tilde{\nu}\left(x, \hat{x}_{1}, s\right)=\tilde{\nu}\left(x, \hat{x}_{2}, s\right)=$ $\langle e, \sigma, \tau\rangle$. We will use $x_{1}$ and $\hat{x}_{1}$ as $x$ and $\hat{x}$ were used above. In addition, we will put $\hat{x}_{2}$ in $\widetilde{T}_{2, s+2}$ and $x_{2}$ in $T_{2, s+1}$. As above, we can show that if $D_{\nu}^{\widetilde{T}_{2}}$ is infinite, then $D_{\nu^{\prime}}^{T_{2}}$ is infinite, for $\nu^{\prime} \geq \nu$. Now if $x_{1}$ is in $T_{2, t}$, for some $t \geq s+1, \nu\left(e, x_{1}, t\right)=\left\langle e, \sigma^{\prime}, \tau\right\rangle$, where $\sigma \subseteq \sigma^{\prime}$, is cocovered by $\tilde{\nu}\left(e, \hat{x}_{2}, s+1\right)=\left\langle e, \sigma, \tau^{\prime}\right\rangle$, where $\tau \subseteq \tau^{\prime}$ (again the matching stops at stage $s)$. If $D_{\nu}^{T_{2}}$ is infinite, then there exists $\nu^{\prime} \leq \nu$ such that $D_{\nu^{\prime}}^{\widetilde{T}_{2}}$ is infinite.

It should be noted that $T_{1}, T_{2},\left\{U_{n}\right\}_{n<\omega}$, and $\left\{\widetilde{V}_{n}\right\}_{n<\omega}$ will live in $\omega$ and $\widetilde{T}_{1}, \widetilde{T}_{2},\left\{\widetilde{U}_{n}\right\}_{n<\omega}$, and $\left\{V_{n}\right\}_{n<\omega}$ in $\hat{\omega}$. We also will have that $T_{1} \cup T_{2}=$ $\widetilde{T}_{1} \cup \widetilde{T}_{2}, \widetilde{T}_{1} \subseteq \widetilde{U}_{0}, T_{1} \subseteq \widetilde{T}_{1}$, and $\widetilde{T}_{2} \subseteq T_{2}$

Construction of $T_{1}, T_{2}, \widetilde{T}_{1}, \widetilde{T}_{2},\left\{\widetilde{U}_{n}\right\}_{n<\omega},\left\{\widetilde{V}_{n}\right\}_{n<\omega}, \Gamma \widetilde{U}_{0}$, and $\gamma(x, s)$.

Stage 0. Let $T_{1,0}=T_{2,0}=\widetilde{T}_{1,0}=\widetilde{T}_{1,1}=\widetilde{T}_{2,0}=\widetilde{T}_{2,1}=\varnothing$ (we will build $\widetilde{T}$ slower than the other sets), $\widetilde{U}_{n, 0}=\widetilde{V}_{n, 0}=\varnothing$ for all $n \in \omega$, and $X_{\nu, 0}=\varnothing$, for all $\nu$. Let $\Gamma_{0}^{\widetilde{U}_{0,0}}(x) \uparrow$ and $\gamma(x, 0) \uparrow$, for all $x$.

Stages +1 . Let $\langle x, e\rangle$ and $\langle\hat{y}, c\rangle$ be such that $x \in U_{e, s+1}-U_{e, s}$ and $\hat{y} \in V_{c, s+1}-V_{c, s}$. For $j \neq e$ and $j \neq 0$, let $\widetilde{U}_{j, s+1}=\widetilde{U}_{j, s}\left(\widetilde{U}_{0}\right.$ will only be built in Step 1, Case d, Step 2 or Step 4) and for $j \neq c$, let $\widetilde{V}_{j, s+1}=\widetilde{V}_{j, s}$.

Step 1. (Defining $\Gamma_{s+1}^{\widetilde{U}_{0}, s+1}(z)$ and $\gamma(z, s+1)$, and building the $\widetilde{T}_{i}$ 's.) For each $z \leq s$, execute the first case that applies.

Case a. If $\gamma(z, s) \uparrow$ and $\left|W_{f(w), s+1} \cap \bar{A}_{s+1}\right|=2^{2 w+2}+2$, for all $w \leq z$, let $\Gamma_{s+1}^{\widetilde{U}_{0, s+1}}(z)=U_{1, s+1}(z)$ and let $\gamma(z, s+1)=\max \left\{\bigcup_{w \leq z} W_{f(w), s+1}\right\}$.

Case b. If $e=0, \gamma(z, s) \downarrow \geq x$ and $\left|W_{f(w), s+1} \cap \bar{A}_{s+1}\right|=2^{2 w+2}+2$ for all $w \leq z$, let $\Gamma_{s+1}^{U_{0}, s+1}(z)=U_{1, s+1}(z)$ and let

$$
\gamma(z, s+1)=\max \left\{\bigcup_{w \leq z} W_{f(w), s+1}\right\} .
$$

(We will put $\hat{x}$ into $\widetilde{U}_{0, s+1}$ in Step 4. Hence this definition of $\gamma(z, s+1)$ is legal.)

Case c. If $e=0, \gamma(z, s) \downarrow \geq x$, and $\left|W_{f(w), s+1} \cap \bar{A}_{s+1}\right| \neq 2^{2 w+2}+2$, for some $w \leq z$, let $\Gamma_{s+1}^{U_{0, s+1}}(z) \uparrow$ and let $\gamma(z, s) \uparrow$. (Again we will put $\hat{x}$ into $\widetilde{U}_{0, s+1}$ in Step 4.) 
Case d. $e=1, z=x$, and $\gamma(z, s) \downarrow$. (In this case, $U_{1}(=K)$ changes, and we are forced to make a change in $\widetilde{U}_{0, s}\lceil\gamma(z, s)$ to copy this change into $\Gamma_{s+1}^{\widetilde{U}_{0, s+1}}(z)$.) Since $\gamma(z, s) \downarrow$, we must have $\mid\left(W_{f(x), s} \cap \bar{A}_{s}\right)\left\lceil\gamma(z, s) \mid \geq 2^{2 x+2}+2\right.$. So there must exist $z_{1}, z_{2} \in W_{f(x), s} \cap \bar{A}_{s}$ such that $z_{i} \neq x$ and $\nu\left(x, z_{1}, s\right)=$ $\nu\left(x, z_{2}, s\right)=\tilde{\nu}\left(x, \hat{z}_{1}, s\right)=\tilde{\nu}\left(x, \hat{z}_{2}, s\right)$. Let $\widetilde{U}_{0, s+1}=\widetilde{U}_{0, s} \cup\left\{\hat{z}_{1}\right\}, \widetilde{T}_{1, s+2}=$ $\widetilde{T}_{1, s+1} \cup\left\{\hat{z}_{1}\right\} \quad\left(\widetilde{T}\right.$ is built slower to meet (1)), and $\widetilde{T}_{2, s+2}=\widetilde{T}_{2, s+1} \cup\left\{\hat{z}_{2}\right\}$ (this helps us meet (2) and (3)). If $\nu \preceq \tilde{\nu}\left(x, \hat{z}_{1}, s+1\right)$, let $X_{\nu, s+1}=X_{\nu, s} \cup\left\{z_{1}\right\}$, otherwise let $X_{\nu, s+1}=X_{\nu, s}$. We will decide (in Step 3) at a later stage whether $z_{1} \in T_{1}$ or $z_{1} \in T_{2}$. We say that $z_{1}$ is delayed. Let $T_{2, s+1}^{\prime}=\left\{z_{2}\right\}$ (we will add this to $T_{2}$ in Step 3). Now $\widetilde{U}_{0, s+1} \backslash \gamma(z, s) \neq \widetilde{U}_{0, s} \backslash \gamma(z, s)$, so we can legally redefine $\Gamma_{s+1}^{U_{0, s+1}}(x)=1$ and $\gamma(x, s+1)=\gamma(x, s)$. After this step is completed, go to Step 3, i.e. skip Step 2.

Case e. Otherwise, let $\Gamma_{s+1}^{\widetilde{U}_{0, s+1}}(z)=\Gamma_{s}^{\widetilde{U}_{0, s+1}}(z)$ and $\gamma(z, s+1)=\gamma(z, s)$. [Note in Step 1 it is essential that $A$ be non-hhsimple. We used the fact that if $\gamma(z, s) \downarrow$ then there are two numbers $z_{1}, z_{2}$ less than $\gamma(z, s)$ such that they both are in $\overline{A_{s} \cup T_{s}}$ and have the same $z$-state. If $A$ is not hhsimple, then it might be impossible to arrange this and have $\lim _{s \rightarrow \omega} \gamma(z, s)<\infty$ (i.e. meet the requirement $\Gamma$ ). For example, if $A$ were maximal and $A \cup T={ }^{*} \omega$.]

Step 2. (Cleanup from Step 1.) Let $T_{2, s+1}^{\prime}=\varnothing, \widetilde{U}_{0, s+1}=\widetilde{U}_{0, s}, \widetilde{T}_{1, s+2}=$ $\widetilde{T}_{1, s+2}=\widetilde{T}_{1, s+1}$, and $\widetilde{T}_{2, s+2}=\widetilde{T}_{2, s+1}$. Let $X_{\nu, s+1}=X_{\nu, s}$, for all $\nu$.

Step 3. (Dealing with the delayed elements and building the $T_{i}$ 's.) For each $z \leq s$, execute the first case that applies. If $z$ is delayed, let $s_{z}$ be the least stage such that $z$ was delayed at stage $s_{z}$, let $k_{z}$ be such that $z \in W_{f\left(k_{z}\right), s}$ (by Step 1, Case d, we know that $k_{z}$ exists and since $W_{f(e)}$ is a weak array $k_{z}$ must be unique), and let $t_{z}$ be such that $z \in W_{g(\nu), t_{z}}$, for all $\nu \preceq \widetilde{\nu}\left(k_{z}, \hat{z}, s_{z}\right)$.

Case a. $z$ is delayed, $s=p\left(t_{z}\right)$, and $z \in A_{s}$. Then we say $z$ is no longer delayed, and let $T_{1, s+1}^{z}=\{z\}$ and $T_{2, s+1}^{z}=\varnothing$.

Case b. $z$ is delayed, $s=p\left(t_{z}\right)$, and $z \notin A_{s}$. Then $z$ is no longer delayed, and let $T_{1, s+1}^{z}=\varnothing$ and $T_{2, s+1}^{z}=\{z\}$.

Case c. Otherwise. Let $T_{1, s+1}^{z}=\varnothing$ and $T_{2, s+1}^{z}=\varnothing$.

Let $T_{1, s+1}=T_{1, s} \cup \bigcup_{z<s} T_{1, s+1}^{z}$ and $T_{2, s+1}=T_{2, s} \cup T_{2, s+1}^{\prime} \cup \bigcup_{z<s} T_{2, s+1}^{z}$. (If $z$ is delayed at stage $s_{z}, t_{z}$ will exist and hence after stage $p\left(t_{z}\right)+1, z$ will no longer be delayed.)

Step 4. (Matching $U_{e}$.) $\{\hat{x}\}$.

Case a. If $x \notin T_{1, s+1} \cup T_{2, s+1}$ and $x$ is not delayed, let $\widetilde{U}_{e, s+1}=\widetilde{U}_{e, s} \cup$

Case b. Otherwise, let $\widetilde{U}_{e, s+1}=\widetilde{U}_{e, s}$.

Step 5. (Matching $V_{c}$.)

Case a. If $\hat{y} \notin \widetilde{T}_{2, s+2} \cup \widetilde{T}_{1, s+2}$, let $\widetilde{V}_{c, s+1}=\widetilde{V}_{c, s} \cup\{y\}$.

Case b. Otherwise, let $\widetilde{V}_{c, s+1}=\widetilde{V}_{c, s}$.

Let $T_{s}=T_{1, s} \cup T_{2, s}$ and $\widetilde{T}_{s}=\widetilde{T}_{1, s} \cup \widetilde{T}_{2, s}$. For any $i$, if $x \in T_{s+1}-T_{s}$, then $x \notin \widetilde{V}_{i, s+1}-\widetilde{V}_{i, s}$ and if $\hat{y} \in \widetilde{T}_{s+1}-\widetilde{T}_{s}$, then $\hat{y} \notin \widetilde{U}_{i, s+1}-\widetilde{U}_{i, s}$ (see 
Step 1, Case $\mathrm{d}$ and Step 2, for $i=0$, and Step 4 and Step 5, for all other $i$ ). Hence condition (1) is met. If we add $x$ to $T$ we also add $\hat{x}$ to $\widetilde{T}$ (see Step 1 , Case d). If we add $\hat{y}$ to $\widetilde{T}$ we also add $y$ to $T$, although sometimes at a later stage (see Step 1, Case d and Step 3). It is easy to see that if $x \notin T$ then $\nu(x, x)=\tilde{\nu}(x, \hat{x})$ (see Step 4 and Step 5). Therefore we meet $R_{\nu}^{0}$. In the next lemma, we show that the requirement $\Gamma$ is met; $B$ is complete. To show $A$ is automorphic to $B$, it is only necessary, at this point, to show conditions (2) and (3) hold. The remaining four lemmas show that conditions (2) and (3) are satisfied.

Lemma 3.7. $\Gamma^{\widetilde{U}_{0}}$ is a functional, $\Gamma^{\widetilde{U}_{0}}=U_{1}, \operatorname{deg}\left(\widetilde{U}_{0}\right) \leq \operatorname{deg}\left(U_{0}\right) \cup \operatorname{deg}\left(U_{1}\right)$, and $\operatorname{deg}(\widetilde{T}) \leq \operatorname{deg}\left(U_{0}\right) \cup \operatorname{deg}\left(U_{1}\right)$. (Hence the requirement $\Gamma$ is met.)

Proof. First $\Gamma^{\widetilde{U}_{0}}=U_{1}$. Let $x \in \omega$. Since $\left|W_{f(i)} \cap \bar{A}\right|=2^{2 i+2}+2$, for all $i$, there exists an $s$ such that for all $y \leq x, W_{f(y), s} \cap \bar{A}_{s}=W_{f(y)} \cap \bar{A}$ and $\left|W_{f(y), s} \cap \bar{A}_{s}\right|=$ $2^{2 y+2}+2$. By Step 1, Case a, $\gamma(y, s) \downarrow$ and for all $t \geq s, \gamma(y, t) \downarrow=\gamma(y, s) \downarrow$. Suppose at stage $t>s, \Gamma_{t}^{\widetilde{U}_{0, t}}(x) \neq \Gamma_{s}^{\widetilde{U}_{0, s}}(x)$, then Step 1, Case d applies at $t$, and hence, $0=\Gamma_{s}^{\widetilde{U}_{0, s}}(x)=U_{1, t-1}(x)=\Gamma_{t-1}^{\widetilde{U}_{0, t-1}}(x) \neq \Gamma_{t}^{\widetilde{U}_{0, t}}(x)=U_{1, t}(x)=1$. Therefore we have that $\Gamma^{\widetilde{U}_{0}}(x)=\lim _{t \rightarrow \infty} \Gamma_{t}^{\widetilde{U}_{0, t}}(x)=U_{1, t}(x)$.

To show that $\operatorname{deg}\left(\widetilde{U}_{0}\right) \leq \operatorname{deg}\left(U_{0}\right) \cup \operatorname{deg}\left(U_{1}\right)$ and $\operatorname{deg}(\widetilde{T}) \leq \operatorname{deg}\left(U_{0}\right) \cup \operatorname{deg}\left(U_{1}\right)$, let $\hat{x} \in \omega$. Using an oracle for $A\left(=U_{0}\right)$ find $s$ such that for all $y \leq x$, $W_{f(y), s} \cap \bar{A}_{s}=W_{f(y)} \cap \bar{A}$ and $A_{s}|x+1=A| x+1$. Now using an oracle for $U_{1}$ find $t \geq s$ such that, $U_{1, t}\left|x+1=U_{1}\right| x+1$. Now we have that, $\hat{x} \in \widetilde{T}$ iff $\hat{x} \in \widetilde{T}_{t+1}$. (Reminder: $W_{f(i)}$ only contains numbers greater than $i$.) If $\hat{x} \notin \widetilde{T}$, then $x \in \widetilde{U}_{0}$ iff $x \in \widetilde{U}_{0, t}$. If $\hat{x} \in \widetilde{T}_{2}$, then $x \notin \widetilde{U}_{0}$. If $\hat{x} \in \widetilde{T}_{1}$, then $x \in \widetilde{U}_{0}$.

Lemma 3.8. $(\forall \nu)\left[D_{\nu}^{\widetilde{T}_{1}}\right.$ is infinite $\Rightarrow\left(\exists \nu^{\prime} \geq \nu\right)\left[D_{\nu^{\prime}}^{T_{1}}\right.$ is infinite $]$.

Proof. Assume $D_{\nu}^{\widetilde{T}_{1}}$ is infinite. Let $e=|\nu|$. So for some $\sigma$ and $\tau$ there are infinitely many $\hat{x}_{i}$ and $s_{i}$ such that $\hat{x}_{i} \in \widetilde{T}_{1, s_{i}}-\widetilde{T}_{1, s_{i}-1}, \nu=\tilde{\nu}\left(e, \hat{x}_{i}, s_{i}-1\right)$, and $\tilde{\nu}\left(e, \hat{x}_{i}, s_{i}-2\right)=\langle e, \sigma, \tau\rangle$. For all $i, x_{i} \in X_{\langle e, \sigma, \tau\rangle, s_{i}-1}$. By the Recursion Theorem, we have that $X_{\langle e, \sigma, \tau\rangle}=W_{g(\langle e, \sigma, \tau\rangle)}$. There exists $t_{i}$ such that $x_{i} \in W_{g(\langle e, \sigma, \tau\rangle), t_{i}}$. Since $A$ is promptly simple, there are infinitely many $i$ such that $x_{i} \in A_{p\left(t_{i}\right)}$. Let $i$ be such that $x_{i} \in A_{p\left(t_{i}\right)}$. In Step 3, all such $x_{i}$ are placed in $T_{1}$ by stage $p\left(t_{i}\right)$. We delayed $x_{i}$ at stage $s_{i}-1$, so we are no longer matching $x_{i}$ and $\hat{x}_{i}$ after stage $s_{i}-2$ (see Step 4 and Step 5). We have $\tilde{\nu}\left(e, \hat{x}_{i}, s_{i}-2\right)=\nu\left(e, x_{i}, s_{i}-2\right)=\langle e, \sigma, \tau\rangle$. Therefore we have the entry state of $\hat{x}_{i}$ is $\tilde{\nu}\left(e, \hat{x}_{i}, s_{i}\right)=\left\langle e, \sigma \cup\{0\}, \tau^{\prime}\right\rangle=\nu$, where $\tau \subseteq \tau^{\prime}$, and the entry state of $x_{i}$ is $\nu\left(e, x_{i}, p\left(t_{i}\right)\right)=\left\langle e, \sigma^{\prime}, \tau\right\rangle$, where $\sigma \cup\{0\} \subseteq \sigma^{\prime}$. There are only finitely many $\sigma^{\prime} \supseteq \sigma \cup\{0\}$. So there must be $\sigma^{\prime} \supseteq \sigma \cup\{0\}$ such that infinitely many of the $x_{i}$ enter $T_{1}$ in state $\left\langle e, \sigma^{\prime}, \tau\right\rangle$. (Note that $\left\langle e, \sigma^{\prime}, \tau\right\rangle \geq\left\langle e, \sigma \cup\{0\}, \tau^{\prime}\right\rangle=\nu$.)

Lemma 3.9. $(\forall \nu)\left[D_{\nu}^{T_{1}}\right.$ is infinite $\Rightarrow\left(\exists \nu^{\prime} \leq \nu\right)\left[D_{\nu^{\prime}}^{\widetilde{T}_{1}}\right.$ is infinite $\left.]\right]$.

Proof. Assume $D_{\nu}^{T_{1}}$ is infinite. Let $e=|\nu|$. So there are infinitely many $x_{i}$ and $t_{i}$ such that $x_{i} \in T_{1, t_{i}}-T_{1, t_{i}-1}$ and $\nu=\nu\left(e, x_{i}, t_{i}\right)$, for $e=|\nu|$. For some $\sigma$ and $\tau$, there are infinitely many $i$ such that there is a stage $s_{i} \leq t_{i}+1$ with 
$\hat{x}_{i} \in \widetilde{T}_{1, s_{i}}-\widetilde{T}_{1, s_{i}-1}$ and $\tilde{\nu}\left(e, \hat{x}_{i}, s_{i}-2\right)=\langle e, \sigma, \tau\rangle$ (if $x \in T_{1}$ then $\hat{x} \in \widetilde{T}_{1}$ ). Let $i$ be such that $\hat{x}_{i} \in \widetilde{T}_{1, s_{i}}-\widetilde{T}_{1, s_{i}-1}$ and $\tilde{\nu}\left(e, \hat{x}_{i}, s_{i}-2\right)=\langle e, \sigma, \tau\rangle$. Since we were matching $x_{i}$ and $\hat{x}_{i}$ up to stage $s_{i}-1$ we have

$$
\tilde{\nu}\left(e, \hat{x}_{i}, s_{i}-2\right)=\nu\left(e, x_{i}, s_{i}-2\right)=\langle e, \sigma, \tau\rangle .
$$

The entry state of $x_{i}$ is $\nu\left(e, x_{i}, t_{i}\right)=\left\langle e, \sigma^{\prime}, \tau\right\rangle=\nu$, where $\sigma \cup\{0\} \subseteq \sigma^{\prime}$ (since $\left.T_{1} \subset A\right)$ and the entry state of $\hat{x}_{i}$ (into $\left.\widetilde{T}_{1}\right)$ is $\tilde{\nu}\left(e, \hat{x}_{i}, s_{i}\right)=\left\langle e, \sigma \cup\{0\}, \tau^{\prime}\right\rangle$, where $\tau \subseteq \tau^{\prime}$. There are only finitely many $\tau^{\prime} \supseteq \tau$. So there must be $\tau^{\prime} \supseteq \tau$ such that infinitely many of the $\hat{x}_{i}$ enter $\widetilde{T}_{1}$ in state $\left\langle e, \sigma \cup\{0\}, \tau^{\prime}\right\rangle$. (Note that $\left\langle e, \sigma \cup\{0\}, \tau^{\prime}\right\rangle \leq\left\langle e, \sigma^{\prime}, \tau\right\rangle=\nu$.)

Lemma 3.10. $(\forall \nu)\left[D_{\nu}^{T_{2}}\right.$ is infinite $\Rightarrow\left(\exists \nu^{\prime} \geq \nu\right)\left[D_{\nu^{\prime}}^{\widetilde{T}_{2}}\right.$ is infinite $\left.]\right]$.

Proof. Assume $D_{\nu}^{\widetilde{T}_{2}}$ is infinite. Let $e=|\nu|$. For some $\sigma$ and $\tau$, there are infinitely many $\hat{x}_{i}$ and $s_{i}$ such that $\hat{x}_{i} \in \widetilde{T}_{2, s_{i}}-\widetilde{T}_{2, s_{i}-1}, \nu=\tilde{\nu}\left(e, \hat{x}_{i}, s_{i}\right)$, and $\tilde{\nu}\left(e, \hat{x}_{i}, s_{i}-2\right)=\langle e, \sigma, \tau\rangle$. We have

$$
\tilde{\nu}\left(e, \hat{x}_{i}, s_{i}-2\right)=\nu\left(e, x_{i}, s_{i}-2\right)=\langle e, \sigma, \tau\rangle .
$$

Therefore we have that the entry state of $\hat{x}_{i}$ is $\tilde{\nu}\left(e, \hat{x}_{i}, s_{i}\right)=\left\langle e, \sigma, \tau^{\prime}\right\rangle=\nu$, where $\tau \subseteq \tau^{\prime}$, and the entry state of $\hat{x}_{i}$ (into $T_{2}$ ) is

$$
\nu\left(e, x_{i}, s_{i}-1\right)=\left\langle e, \sigma^{\prime}, \tau\right\rangle,
$$

where $\sigma \subseteq \sigma^{\prime}$. There are only finitely many $\sigma^{\prime} \supseteq \sigma$. So there must be $\sigma^{\prime} \supseteq \sigma$ such that infinitely many of the $x_{i}$ enter $T_{2}$ in state $\left\langle e, \sigma^{\prime}, \tau\right\rangle$. (Note that $\left\langle e, \sigma^{\prime}, \tau\right\rangle \geq\left\langle e, \sigma, \tau^{\prime}\right\rangle=\nu$.)

Lemma 3.11. $(\forall \nu)\left[D_{\nu}^{T_{2}}\right.$ is infinite $\Rightarrow\left(\exists \nu^{\prime} \leq \nu\right)\left[D_{\nu^{\prime}}^{\widetilde{T}_{2}}\right.$ is infinite $\left.]\right]$.

Proof. Assume $D_{\nu}^{T_{2}}$ is infinite. Let $e=|\nu|$. So there are infinitely many $x_{i}$ and $t_{i}$ such that $x_{i} \in T_{2, t_{i}}-T_{2, t_{i}-1}$ and $\nu=\nu\left(e, x_{i}, t_{i}\right)$, for $e=|\nu|$. For some $\sigma$ and $\tau$, there are infinitely many $i$ such that there is a stage $s_{i} \leq t_{i}+1$ with $\hat{x}_{i} \in \widetilde{T}_{s_{i}}-\widetilde{T}_{s_{i}-1}$ and $\tilde{\nu}\left(e, \hat{x}_{i}, s_{i}-2\right)=\langle e, \sigma, \tau\rangle$. Let $i$ be such that $\hat{x}_{i} \in \widetilde{T}_{s_{i}}-\widetilde{T}_{s_{i}-1}$ and $\tilde{\nu}\left(e, \hat{x}_{i}, s_{i}-2\right)=\langle e, \sigma, \tau\rangle$. If $\hat{x}_{i} \notin \widetilde{T}_{2}$, then there exists an $\hat{x}_{i}^{\prime} \in \widetilde{T}_{2, s_{i}}-\widetilde{T}_{2, s_{i}-1}$ such that $\tilde{\nu}\left(e, \hat{x}_{i}, s_{i}-2\right)=\nu\left(e, \hat{x}_{i}^{\prime}, s_{i}-2\right)=\langle e, \sigma, \tau\rangle$ (see Step 1, Case d). If $\hat{x}_{i} \in \widetilde{T}_{2}$, let $\hat{x}_{i}^{\prime}=\hat{x}_{i}$. We have $\tilde{\nu}\left(e, \hat{x}_{i}^{\prime}, s_{i}-2\right)=$ $\nu\left(e, x_{i}, s_{i}-2\right)=\langle e, \sigma, \tau\rangle$. Therefore we have that the entry state of $x_{i}$ is $\nu\left(e, x_{i}, t_{i}\right)=\left\langle e, \sigma^{\prime}, \tau\right\rangle$, where $\sigma \subseteq \sigma^{\prime}$ and the entry state of $\hat{x}_{i}^{\prime}$ (into $\widetilde{T}_{2}$ ) is $\tilde{\nu}\left(e, \hat{x}_{i}^{\prime}, s_{i}\right)=\left\langle e, \sigma, \tau^{\prime}\right\rangle$, where $\tau \subseteq \tau^{\prime}$. There are only finitely many $\tau^{\prime} \supseteq \tau$. So there must be a $\tau^{\prime} \supseteq \tau$ such that infinitely many of the $\hat{x}_{i}^{\prime}$ enter $\widetilde{T}_{2}$ in state $\left\langle e, \sigma, \tau^{\prime}\right\rangle$. (Note $\left\langle e, \sigma, \tau^{\prime}\right\rangle \leq\left\langle e, \sigma^{\prime}, \tau\right\rangle$.)

It can be seen that the above automorphism is effective.

If we look carefully at the above proof, we can see that, in fact, we have proven the following theorem.

Theorem 3.12. Let $U_{0}$ be a coinfinite non-hhsimple set. Let $\left\{U_{n, s}\right\}_{n, s<\omega}$ and $\left\{V_{n, s}\right\}_{n, s<\omega}$ be simultaneous enumerations of all the r.e. sets. $\left(U_{1}\right.$ can be anything.) Then there exist r.e. sets $T, \widetilde{T}_{1}, \widetilde{T}_{2},\left\{\widetilde{V}_{n}\right\}_{n<\omega},\left\{\widetilde{U}_{n}\right\}_{n<\omega}$ and a simultaneous enumeration of all these r.e. sets such that: $\widetilde{T}_{1}$ and $\widetilde{T}_{2}$ are disjoint, $\widetilde{T}_{1}=\left(\widetilde{U}_{0} \searrow\left(\widetilde{T}_{1} \cup \widetilde{T}_{2}\right)\right)$, and

$$
\operatorname{deg}\left(U_{1}\right) \leq \operatorname{deg}\left(\widetilde{U}_{0}\right)
$$




$$
\operatorname{deg}\left(\widetilde{U}_{0}\right) \leq \operatorname{deg}\left(U_{0}\right) \cup \operatorname{deg}\left(U_{1}\right)
$$

$$
\begin{gathered}
\operatorname{deg}\left(\widetilde{T}_{1} \cup \widetilde{T}_{2}\right) \leq \operatorname{deg}\left(U_{0}\right) \cup \operatorname{deg}\left(U_{1}\right), \\
\exists^{\infty} x \in \bar{T} \text { with final e-state } \nu \text { w.r.t. to }\left\{U_{n, s}\right\}_{n, s<\omega} \text { and }\left\{\widetilde{V}_{n, s}\right\}_{n, s<\omega}
\end{gathered}
$$

$\exists^{\infty} \hat{x} \in \overline{\left(\widetilde{T}_{1} \cup \widetilde{T}_{2}\right)}$ with final e-state $\nu$ w.r.t. to $\left\{\widetilde{U}_{n, s}\right\}_{n, s<\omega}$ and $\left\{V_{n, s}\right\}_{n, s<\omega}$,

$$
\begin{gathered}
\forall n\left[T \searrow \widetilde{V}_{n}=\left(\widetilde{T}_{1} \cup \widetilde{T}_{2}\right) \searrow \widetilde{U}_{n}=\varnothing\right], \\
(\forall \nu)\left[D_{\nu}^{\widetilde{T}_{2}} \text { is infinite } \Rightarrow\left(\exists \nu^{\prime} \geq \nu\right)\left[D_{\nu^{\prime}}^{T} \text { is infinite }\right]\right] \\
(\forall \nu)\left[D_{\nu}^{T} \text { is infinite } \Rightarrow\left(\exists \nu^{\prime} \leq \nu\right)\left[D_{\nu^{\prime}}^{\widetilde{T}_{2}} \text { is infinite }\right]\right] .
\end{gathered}
$$

Furthermore, if $U_{0}$ is promptly simple then, in addition, we have

$$
(\forall \nu)\left[D_{\nu}^{\widetilde{T}_{1}} \text { is infinite } \Rightarrow\left(\exists \nu^{\prime} \geq \nu\right)\left[D_{\nu^{\prime}}^{T} \text { is infinite }\right]\right] .
$$

Note that (7) is the same as $R_{\nu}$ and conditions (8)-(10) are the same as the conditions (1) $-(3)$ in the hypotheses of the Extension Theorem. In the above construction, we clearly proved this in the case when $U_{0}$ is promptly simple. If $U_{0}$ is not promptly simple, the only change we need is that instead of delaying elements, we will place them into $T_{2}$ (hence $T_{1}=\varnothing$ ). This makes Step 1, Case d simpler since we no longer need the $X_{\nu}$ 's and, in addition, we no longer need Step 3. Lemmas 3.8 and 3.9 are not needed and Lemmas 3.10 and 3.11 can remain almost unchanged. We will leave the rest of details to the reader.

In the proof of Theorem 3.3 we applied the Extension Theorem to extend the isomorphism formed by Theorem $3.12, \mathscr{L}^{*}(T) \approx \mathscr{L}^{*}\left(\widetilde{T}_{1} \cup \widetilde{T}_{2}\right)$, into an automorphism of $\mathscr{E}^{*}$, and to get $U_{0} \approx \widehat{U}_{0}(A \approx B)$. If $U_{0}$ is not promptly simple, then at least we can apply the Extension Theorem to show $\mathscr{L}^{*}\left(U_{0}\right) \approx$ $\mathscr{L}^{*}\left(\widetilde{U}_{0}\right)$. (We extend the isomorphism, $\mathscr{L}^{*}(T) \approx \mathscr{L}^{*}\left(\widetilde{T}_{1} \cup \widetilde{T}_{2}\right)$, to include $\mathscr{E}^{*}(T) \approx \mathscr{E}^{*}\left(\widetilde{T}_{2}\right)$. Now since $\widetilde{T}_{1} \subseteq \widetilde{U}_{0}$ we have $\mathscr{L}^{*}\left(U_{0}\right) \approx \mathscr{L}^{*}\left(\widetilde{U}_{0}\right)$ by this extended isomorphism.) This gives us a more pleasing proof of Theorem 2.4 in the case that the given set $A$ is not hhsimple (again let $U_{1}=K$ ).

By directly applying Theorems 3.2 and 3.3, as follows, we can also prove Theorem 2.4. This proof is due to Todd Hammond.

Another proof of Theorem 2.4 (Hammond). Let $P$ be a low promptly simple set. By Soare [1982], we know that $\mathscr{E}^{*} \approx_{\text {eff }} \mathscr{L}^{*}(P)$ by some isomorphism $\Phi$. Given any coinfinite r.e. set $B$, let $D=\Phi(B) \cup P$. Therefore, $\mathscr{L}^{*}(B) \approx \mathscr{L}^{*}(D)$ and $D$ is also coinfinite. Since the promptly simple sets form a filter in $\mathscr{E}$ (Maass, Shore, and Stob [1981]), $D$ is promptly simple. Hence there is a complete set $C$ such that $\mathscr{L}^{*}(C) \approx \mathscr{L}^{*}(D)$.

\section{FinAL REMARKS}

Using these methods, it is not possible to build the above $B$ (where $A \approx$ $B$ for $A$ non-hhsimple promptly simple or $\mathscr{L}^{*}(A) \approx \mathscr{L}^{*}(B)$ for $A$ nonhhsimple) such that $\operatorname{deg}(B)=\mathbf{d}$, for any degree d. As we noted before $\widetilde{U}_{0}$ 
and $T_{2} \searrow \widehat{U}_{0}\left(T_{1} \subseteq \widetilde{U}_{0}\right)$ is a splitting of $B$. We have some degree-theoretic control over $\widehat{U}_{0}$; we can make $\operatorname{deg}\left(\widetilde{U}_{0}\right) \leq \operatorname{deg}(A) \cup \mathbf{d}$. But if $\hat{x} \in \widetilde{T}_{2}$, the Extension Theorem has control over whether $\hat{x}$ is put in $\hat{U}_{0}$. In the current form of the Extension Theorem, the set $T \searrow \widehat{U}_{0}$ may have arbitrary degree; we have no degree-theoretic control over $T \searrow \widehat{U}_{0}$. In his thesis, Cholak produces a more degree-theoretic version of the Extension Theorem, the "High Extension Theorem," which he uses along with Theorem 3.13 to show that for all r.e. high degrees $\mathbf{h}$ and for all coinfinite r.e. sets $A$ there exists an r.e. set $B \in \mathbf{h}$ such that $\mathscr{L}^{*}(A) \approx \mathscr{L}^{*}(B)$.

Harrington and Soare have recently completed a uniform proof that every r.e. set of promptly simple degree is effectively automorphic to a complete set.

Theorem 4.1 (Harrington and Soare [1991]). If $A$ is an r.e. set of promptly simple degree then there is an effective automorphism $\Phi$ of the lattice of r.e. sets such that $\Phi(A)$ is complete. Furthermore, $\Phi$ can be found uniformly effectively from an index of $A$ and an index for the recursive function witnessing that $A$ has promptly simple degree.

Hence, if $A$ is not automorphic to a complete set, $A$ cannot have promptly simple degree. We still, however, are lacking a complete characterization of the r.e. sets which are automorphic (or effectively automorphic) to a complete set.

\section{REFERENCES}

1. P. Cholak, Automorphisms of the lattice of recursively enumerable sets (in preparation).

2. _ Automorphisms of the lattice of recursively enumerable sets, Ph.D. Dissertation, Univ. of Wisconsin, 1991.

3. J.C.E. Dekker and J. Myhill, Retraceable sets, Canad. J. Math 10 (1958), 357-373.

4. R. G. Downey and M. Stob, Automorphisms of the lattice of recursively enumerable sets: Orbits, Adv. in Math. (to appear).

5. L. Harrington and R. Soare, Post's program and incomplete recursively enumerable sets, Proc. Nat. Acad. Sci. U.S.A. 88 (1991), 10242-10246.

6. C. G. Jockusch, Uniformly introreducible sets, J. Symbolic Logic 33 (1968), 521-536.

7. A. H. Lachlan, On the lattice of recursively enumerable sets, Trans. Amer. Math. Soc. 130 (1968), 1-37.

8. W. Maass, Characterization of recursively enumerable sets with supersets effectively isomorphic to all recursively enumerable sets, Trans. Amer. Math. Soc. 279 (1983), 311-336.

9. __ Orbits of hyperhypersimple sets, J. Symbolic Logic 49 (1984), 51-62.

10. W. Maass, R. A. Shore, and M. Stob, Splitting properties and jump classes, Israel J. Math. 39 (1981), 210-224.

11. S. S. Marchenkov, A class of incomplete sets, Mat. Zametki 20 (1976), 473-478; English transl., Math Notes 20 (1976), 823-825.

12. D. A. Martin, Classes of recursively enumerable sets and degrees of unsolvability, Z. Math. Logik Grundlag. Math. 12 (1966), 295-310.

13. D. Miller, The relationship between the structure and degrees of recursively enumerable sets, Ph.D. Dissertation, Univ. of Chicago, 1981.

13. P. Odifreddi, Classical recursion theory, North-Holland, Amsterdam, New York, and Oxford, 1989.

14. R. Soare, Automorphisms of the lattice of recursively enumerable sets, Part I: Maximal sets, Ann. of Math. (2) 100 (1974), 80-120.

15. __ Automorphisms of the lattice of recursively enumerable sets, Part II: Low sets, Ann. of Math. Logic 22 (1982), 69-107. 
16. _ـ Recursively enumerable sets and degrees, Perspectives in Mathematical Logic, Omega Series, Springer-Verlag, Berlin, Heidelberg, New York, 1987.

17. C. E. M. Yates, Three theorems on the degree of recursively enumerable sets, Duke Math. J. 32 (1965), 461-468.

Department of Mathematics, University of Wisconsin, Madison, Wisconsin 53706

Current address: Department of Mathematics, University of Michigan, Ann Arbor, Michigan 48109-1003

E-mail address: cholak@math.lsa.umich.edu

Department of Mathematics, Victoria University of Wellington, P.O. Box 600, WelLINGTON, NeW ZeALAND

E-mail address: downey@math.vuw.ac.nz

Department of Mathematics, Calvin College, Grand Rapids, Michigan 49546

E-mail address: stob@calvin.edu 